\title{
Effect of Lean Processes on Surgical Wait Times and Efficiency in a Tertiary Care Veterans Affairs Medical Center
}

Nakul P. Valsangkar, MD; Andrew C. Eppstein, MD; Rick A. Lawson, BSEE; Amber N. Taylor, MHA

IMPORTANCE There are an increasing number of veterans in the United States, and the current delay and wait times prevent Veterans Affairs institutions from fully meeting the needs of current and former service members. Concrete strategies to improve throughput at these facilities have been sparse.

OBJECTIVE To identify whether lean processes can be used to improve wait times for surgical procedures in Veterans Affairs hospitals.

DESIGN, SETTING, AND PARTICIPANTS Databases in the Veterans Integrated Service Network 11 Data Warehouse, Veterans Health Administration Support Service Center, and Veterans Information Systems and Technology Architecture/Dynamic Host Configuration Protocol were queried to assess changes in wait times for elective general surgical procedures and clinical volume before, during, and after implementation of lean processes over 3 fiscal years (FYs) at a tertiary care Veterans Affairs medical center. All patients evaluated by the general surgery department through outpatient clinics, clinical video teleconferencing, and e-consultations from October 2011 through September 2014 were included. Patients evaluated through the emergency department or as inpatient consults were excluded.

EXPOSURES The surgery service and systems redesign service held a value stream analysis in FY 2013, culminating in multiple rapid process improvement workshops. Multidisciplinary teams identified systemic inefficiencies and strategies to improve interdepartmental and patient communication to reduce canceled consultations and cases, diagnostic rework, and no-shows. High-priority triage with enhanced operating room flexibility was instituted to reduce scheduling wait times. General surgery department pilot projects were then implemented mid-FY 2013.

MAIN OUTCOMES AND MEASURES Planned outcome measures included wait time, clinic and telehealth volume, number of no-shows, and operative volume. Paired $t$ tests were used to identify differences in outcome measures after the institution of reforms.

RESULTS Following rapid process improvement workshop project rollouts, mean (SD) patient wait times for elective general surgical procedures decreased from 33.4 (8.3) days in FY 2012 to 26.0 (9.5) days in FY 2013 ( $P=.02$ ). In FY 2014, mean (SD) wait times were half the value of the previous FY at $12.0(2.1)$ days $(P=.07)$. This was a 3 -fold decrease from wait times in $\mathrm{FY}$ $2012(P=.02)$. Operative volume increased from 931 patients in FY 2012 to 1090 in FY 2013 and 1072 in FY 2014. Combined clinic, telehealth, and e-consultation encounters increased from 3131 in FY 2012 to 3460 in FY 2013 and 3517 in FY 2014, while the number of no-shows decreased from 366 in FY 2012 to 227 in FY $2014(P=.02)$.

CONCLUSIONS AND RELEVANCE Improvement in the overall surgical patient experience can stem from multidisciplinary collaboration among systems redesign personnel, clinicians, and surgical staff to reduce systemic inefficiencies. Monitoring and follow-up of system efficiency measures and the employment of lean practices and process improvements can have positive short- and long-term effects on wait times, clinical throughput, and patient care and satisfaction.

JAMA Surg. 2017;152(1):42-47. doi:10.1001/jamasurg.2016.2808

Published online September 7, 2016
Invited Commentary page 48

Author Affiliations: Section of General Surgery, Department of Surgery, Indiana University School of Medicine, Indianapolis (Valsangkar Eppstein); Surgery Service, Richard L. Roudebush Veterans Affairs Medical Center, Indianapolis, Indiana (Eppstein, Taylor); Systems Redesign Service, Richard L. Roudebush Veterans Affairs Medical Center, Indianapolis, Indiana (Lawson).

Corresponding Author: Andrew C. Eppstein, MD, Richard L. Roudebush Veterans Affairs Medical Center, 1481 W Tenth St, Surgery Service 112, Indianapolis, IN 46202 (aeppstei@iupui.edu). 
$\mathrm{T}$ he Veterans Health Administration (VHA) is the largest integrated health care network in the United States, providing a unique system of health care delivery and access to 9 million veterans. ${ }^{1}$ However, it has come under increased media scrutiny over the past 2 years for delays in scheduling, lengthy patient wait times, and lack of access. ${ }^{1-8}$ The US Department of Veterans Affairs (VA) has seen a steady increase in the number of enrollees and use of health care services over the past decade, ${ }^{1,2}$ causing strain on the existing systems, with increasing demand from aging Vietnam war-era veterans as well as younger veterans from recent conflicts. Outrage over unacceptable patient wait times and access problems prompted the passage of the Veterans Access, Choice, and Accountability Act of 2014, ${ }^{9-11}$ which formed the Commission on Care to evaluate the way in which the VA provides care and make short- and long-term recommendations to increase effectiveness and efficiency within the system. This commission's report in September $2015^{12}$ found a significant gap between demand and product delivered as well as uneven operations and processes. ${ }^{3}$

The Richard L. Roudebush Veterans Affairs Medical Center is a busy tertiary care referral center within the VA, serving more than 60000 patients with a catchment radius of 200 miles. By fiscal year (FY) 2012, increasing patient wait times for surgical procedures prompted collaboration between the surgery service and systems redesign service to improve wait times, reduce systemic inefficiencies, and improve patient satisfaction through the enactment of lean processes. A multidisciplinary group composed of surgeons, nurses, operating room (OR) staff, administration, and lean experts met to conduct a value stream analysis (VSA) in 2013, which identified inefficiencies and formulated strategies to improve interdepartmental and patient communication as well as to reduce canceled consultations and cases, diagnostic reworks, and clinical no-shows, with the goal of enhancing patient access and reducing wait times. The VSA identified multiple problem areas that could be addressed through smaller committees and projects as well as metrics to assess the results of these projects. The general surgery department was selected to roll out pilot reforms, which were implemented in mid-FY 2013.

The objective of this study was to evaluate the effectiveness of these pilot projects at decreasing patient wait times and improving scheduling efficiency and access. We also critically examined the processes used in this project to make such a process replicable in VA facilities across the country.

\section{Methods}

A committee comprised of representatives from the surgery service, including surgeons, case managers, OR leadership, and sterile processing personnel, began mapping a value stream with guidance from systems redesign staff in January 2013. The current state of patient care was diagrammed from referral to scheduling, operation, perioperative care, follow-up, and discharge from surgical clinics. Inefficiencies, delays, communication breakdowns, and rework were mapped at multiple points of the value stream map to identify waste and areas for

\section{Key Points}

Question Can lean processes be used to improve patient wait times for surgical procedures in Veterans Affairs hospitals?

Findings In this systematic review of institutional wait list data from fiscal years 2012 to 2014, the implementation of lean system redesigns was associated with a significant and sustained wait list reduction from 33.4 days to 12.0 days for patients waiting for elective general surgical procedures.

Meaning Multidisciplinary system redesigns using lean principles may decrease patient wait times by addressing and correcting systemic inefficiencies.

improvement. The committee then diagrammed a lean, idealized version for the anticipated future state and what would be needed to achieve it.

Per VHA policy, Handbook 1200.05, Appendix A, the article presents information that involves the collection or study of existing deidentified data and therefore does not require informed consent or institutional review board approval.

\section{Primary Areas of Improvement for Rapid Experiments} Specific improvements included standardizing preoperative anesthesia referrals and triage of patients for surgical procedure scheduling, restructuring consultation methods and service agreements, improving interdepartmental communication, increasing patient communication, facilitating transportation and lodging for distant patients, and standardizing work across surgical subspecialties (Figure 1). Improvements in these fields were assessed for ease in implementation and time required to complete and were classified as "just do its" (JDIs), rapid process improvement workshops (RPIWs), or projects for simple, mid-range, and complex solutions. Hypotheses were generated regarding the effect of improvement on wait time, cost, patient/staff satisfaction, and organizational efficiency.

Figure 1. Methods Used During the Implementation of General Surgery Department Pilot

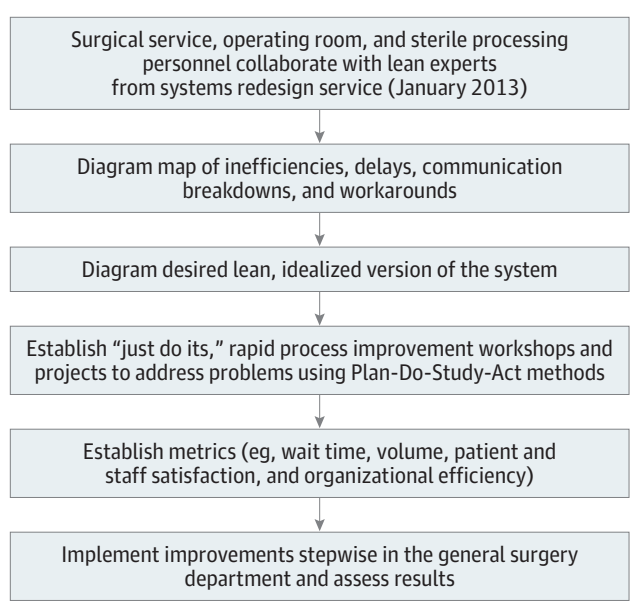




\begin{tabular}{|c|c|c|c|}
\hline Metric & Expanded Metrics & Baseline Value $^{\mathrm{a}}$ & Goal, \% \\
\hline \multirow[t]{4}{*}{ Consultations } & Consultations canceled, \% & 24 & -25 \\
\hline & Consultations scheduled or completed, No. & 17634 & +25 \\
\hline & E-consultations, No. & 50 & +20 \\
\hline & Telemedicine encounters, No. & $155^{\mathrm{b}}$ & +25 \\
\hline \multirow[t]{5}{*}{ Clinic appointment } & Estimated patients seen in $<14 \mathrm{~d}$, $\%$ & 93 & 98 \\
\hline & Missed opportunity rate, $\%$ & 12 & 10 \\
\hline & Patients waiting to be seen, No. & 7633 & -20 \\
\hline & Clinic appointment no-shows, No. & 366 & -10 \\
\hline & Avoidable canceled OR appointments, No. & 1875 & -20 \\
\hline \multirow[t]{4}{*}{ OR use } & Delay in OR scheduling, $d$ & 65 & -25 \\
\hline & Patients waiting to be scheduled for OR, No. & 493 & -25 \\
\hline & Avoidable cancelation rate, \% & 12 & -20 \\
\hline & Mean block use for each service, $\%$ & 83 & 80 \\
\hline \multirow[t]{5}{*}{ Discharge } & Anticipated discharge appointments by noon, $\%$ & 94.8 & 95 \\
\hline & Patients discharged by noon, \% & 59.5 & 80 \\
\hline & Patients discharged within 60 min of discharge order, $\%$ & 17.1 & 30 \\
\hline & Admission criteria met, $\%$ & 92 & 95 \\
\hline & Continuous stay met, \% & 80 & 95 \\
\hline
\end{tabular}

Abbreviations: FY, fiscal year; OR, operating room.

a Baseline values calculated for $\mathrm{FY}$ 2012.

b The baseline value for telemedicine encounters was calculated for $\mathrm{FY}$ 2013.
Targeted Metrics of Improvement

Through the VSA, 4 crucial areas of improvement were found to be consultations, clinic appointments, OR use, and discharge events (Table). These were further broken down into subcategories. Furthermore, baseline parameters were identified for each of these metrics using databases in the Veterans Integrated Service Network 11 Data Warehouse, VHA Support Service Center, and Veterans Information Systems and Technology Architecture/Dynamic Host Configuration Protocol. For example, the baseline for percentage of canceled consultations was $24 \%$, and a $25 \%$ decrease was established as the goal.

\section{Rapid Experimentation Projects}

The VSA generated numerous JDIs and RPIWs between February and June 2013. Service agreements between the general surgery department and referring services were revised with computerized consultation templates outlining necessary preoperative workup to reduce diagnostic rework. Telehealth remote clinic visits and e-consultations were used to process patients prior to clinic visits. The division of labor for each team member (eg, case managers and medical support assistants) in the clinic was made consistent. No-show policies were also standardized. Changes to the process of scheduling surgical procedures involved having referrals complete computerized templates to ensure all necessary patient information was included before coming in for preadmission testing or the OR. Patients were tentatively scheduled using a webbased visual calendar rather than the existing text-based scheduling system to allow more flexibility and were only entered into the scheduling system 1 to 2 weeks prior to their surgical procedure. Additional telephone reminders for patient appointments were enacted within a week of the clinic or operative date to decrease no-shows and facilitate rescheduling. The OR enacted several RPIWs to increase the flexibility of surgical block time, especially for high-priority patients, and to create a liaison between sterile processing and OR personnel to eliminate equipment lags.

The general surgery department, being the most active surgical service, was chosen to pilot the projects enacted by the VSA. Improvements learned in JDIs were implemented immediately, while more complex reforms from RPIWs and projects were rolled out stepwise. Committees continued to meet to review ongoing metrics, and projects were closed once goals had been achieved. Multiple databases were queried for analyses of outcomes per established metrics. Wait times for the general surgery department from clinic evaluation to surgical procedure were drawn from the Veterans Integrated Service Network 11 Data Warehouse. The VHA Support Service Center supplied clinic volume and no-show data. E-consultation, telehealth, and operative volume data were gathered by querying Veterans Information Systems and Technology Architecture/Dynamic Host Configuration Protocol. The period of FY 2012 through FY 2014 was selected because surgical staffing remained stable throughout this period.

\section{Statistical Analysis}

All variables were categorized as either categorical or continuous. All data were calculated using Microsoft Excel 2015 and SPSS Statistics version 15 (SPSS Inc). Continuous variables were compared using $t$ test of means when 2 variables were compared or analysis of variables when more than 2 variables were compared. Categorical variables were compared using the $\mathrm{X}^{2}$ test. Statistical significance was set at $P<.05$.

\section{Results}

In FY 2012, mean (SD) patient wait times for elective general surgical procedures was 33.4 (8.3) days. In the first 8 months of FY 
2013, mean wait times continued to be stable until a statistically significant drop in the last 4 months of FY 2013, which brought the mean (SD) time to 26.0 (9.5) days for all of FY $2013(P=.02)$. This decrease appeared to coincide with the rollout of multiple RPIWs. The trend for decreased wait times continued to hold through FY 2014 at $12.0(2.1)$ days $(P=.07)$, half of the previous fiscal year and 3-fold less than FY $2012(P=.02)$ (Figure 2).

Total operative volume through the general surgery department increased from 931 patients in FY 2012 to 1090 in FY 2013 and 1072 in FY 2014, with no changes in surgeons or patient mix, despite the closure of one OR in early 2014 (Figure 3A). While clinic volume fluctuated from 3131 visits in FY 2012 to 3241 in FY 2013, decreasing to 3084 visits in FY 2014 (Figure 3B), this was offset by increased use of telehealth approaches, including e-consultations (Figure 3C), where medical record review is used to answer a specific question without necessitating a clinic visit, and clinical video teleconferencing (CVT) (Figure 3D). E-consultations rose from 50 in FY 2012 to 64 in FY 2013 to 129 in FY 2014. Clinical video teleconferencing visits, which were not available in FY 2012, rose from 155 visits in FY 2013 to 304 in FY 2014.

Thus, combined clinic, CVT, and e-consultation encounters increased from 3131 in FY 2012 to 3460 in FY 2013 and 3517 in FY 2014. Despite the increased number of patients seen, noshows decreased from 366 in FY 2012 and 346 in FY 2013 to 227 in FY $2014(P=.02)$ (Figure 4).

\section{Discussion}

This study demonstrated a significant reduction in patient wait times for surgical procedures and an improvement in access in the clinical and operative settings when implementing lean processes. The improvement gained was noted over multiple areas and seen during the implementation of new technologies. The changes in the measured outcome categories occurred early, and the differences were sustained across the entire observation period.

Lean methods were first pioneered by Womack and Jones ${ }^{13}$ in the 1980s as part of Toyota's manufacturing processes and have been used increasingly in the health care setting to eliminate waste and improve value. ${ }^{14-20}$ Lean processes, as defined by the Lean Institute, are designed to maximize the value provided to the customer with minimal waste. Other key principles of the lean system are reducing waste throughout the entire value stream, reducing the time and resources required to generate services for customers, and enhancing the system's ability to respond to changes in the needs of the customer with fast throughput times. ${ }^{13,21}$ The VA system is fertile ground to benefit from the application of all of these principles. Budgetary restrictions in government spending toward the VA make low-cost but highly effective health care delivery a high priority for the VA. The high volume of patients seen by the VA in recent times coupled with a need to adapt to the widely varying "customer" volume correspond with lean principles. ${ }^{22}$ Given its pioneering role in electronic medical record systems as well as the national connectivity between all of the different VA systems, ${ }^{23}$ the application and execution of lean principles may be ideal for improving the ef-
Figure 2. Average Surgical Wait Time for General Surgery Department Patients

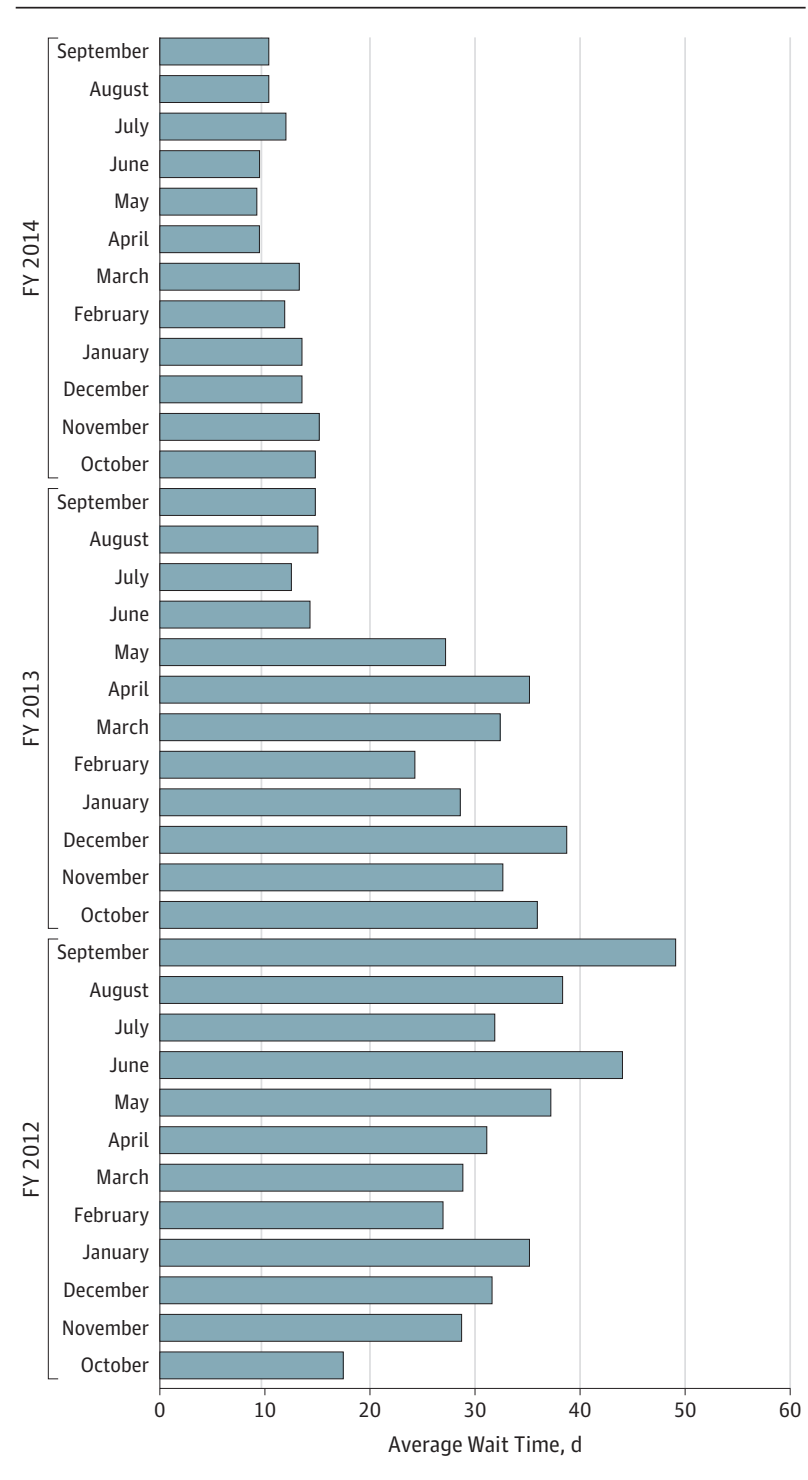

The average wait time for patients decreased from the time of the implementation of the value stream analysis. These differences were statistically significant. The mean (SD) wait times in fiscal year (FY) 2012 were 33.4 (8.3) days, 26.0 (9.5) days in FY 2013 ( $P=.02$ compared with FY 2012), and 12.0 (2.1) days in FY 2014 ( $P=.02$ compared with FY 2012).

ficiency of the VA system. With patient wait times in the VA achieving national prominence, lean methods may be helpful in reducing backlogs and streamlining inefficiencies in the VA system, as they have done in other areas of health care. ${ }^{17-19,24,25}$

This study demonstrated that there were improvements in the volume of general surgery patients seen both in the clinic and the OR. Part of this improvement involves the adoption of e-consultations, which can be used as a formal form of communication between physicians, residents, and nurse practitioners within the electronic medical record. Primary care physicians often use e-consultations for focused questions or so specialists can answer a question that does not require direct patient interaction. In addition to the time flexibility for the 


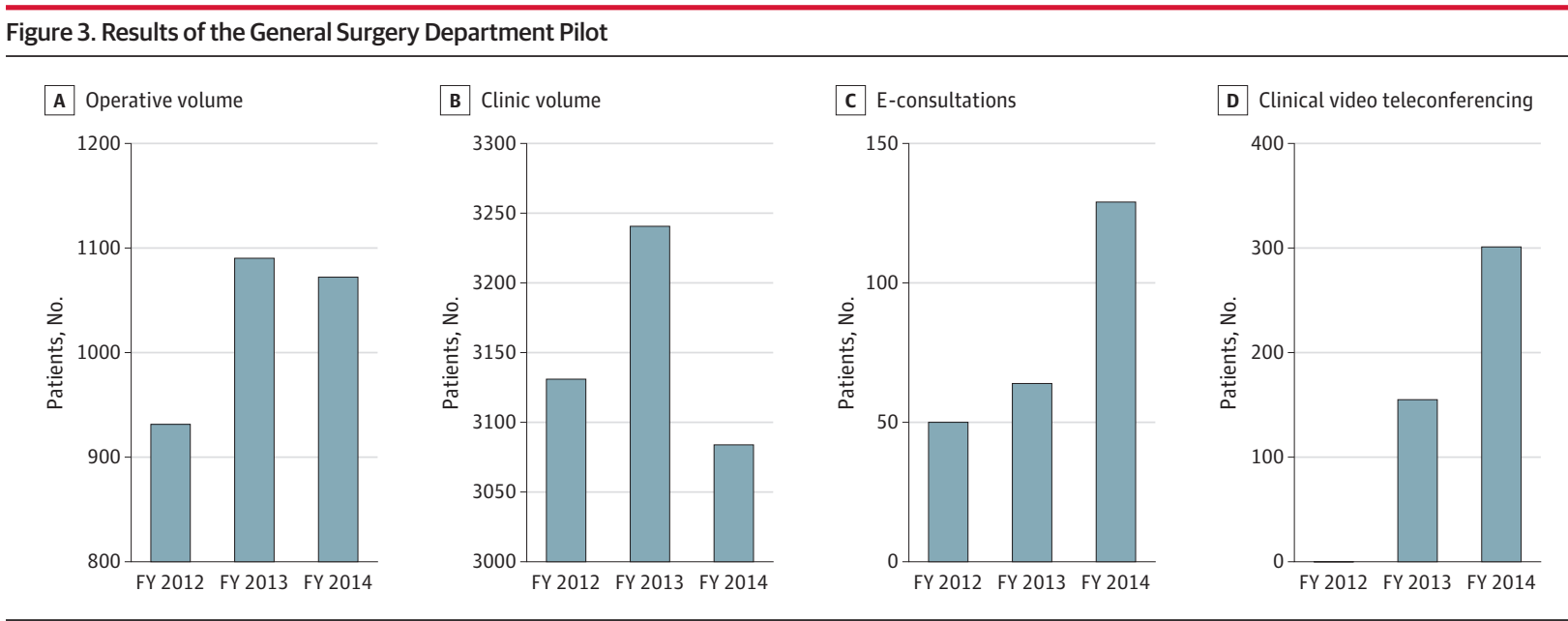

Clinical video teleconferencing was not previously available in fiscal year (FY) 2012.

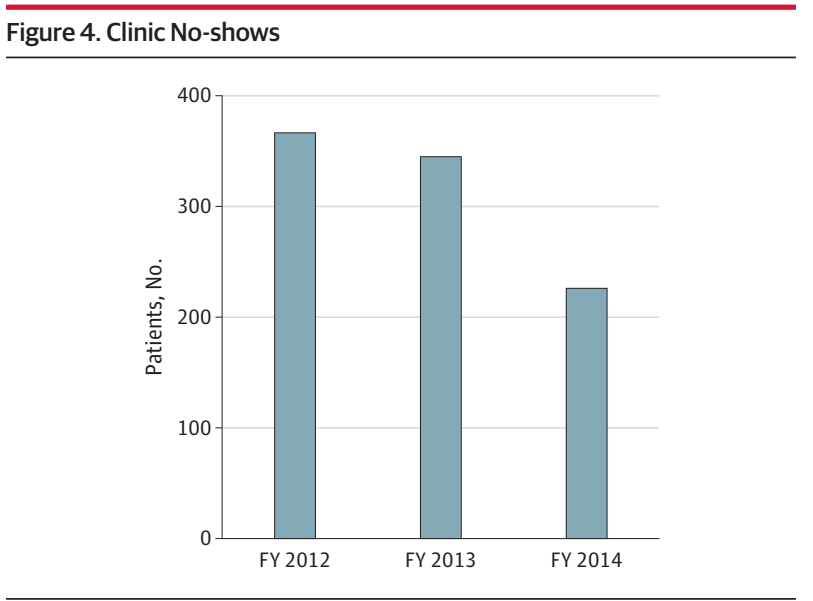

parties involved, there is also a well-defined turnaround time of 72 hours. This, along with a decreased need for travel for straightforward questions, significantly decreases the backlog of wait times and improves patient satisfaction.

This study also demonstrated that there was significant improvement in the use of CVT. ${ }^{26}$ The VA system pioneered the implementation of this technology, and it is used for pre- and postoperative assessments and patient education. ${ }^{26-28}$ Ostensibly, this supports reducing wait times, given that it eliminates a large number of unnecessary clinic visits and travel times. A recent audit of the system demonstrated that there were missed opportunities to capitalize on the available infrastructure and only a limited annual growth (13\%) of noninstitutional care patients using CVT. ${ }^{27}$ The improvement in the e-consultation and CVT visits shown in this study were much more dramatic, and between FY 2013 and FY 2014, there was a 100\% increase in the number of patients seen on CVT. Clinical video teleconference visits were added to existing clinic days, obviating the need for a separate clinic. A combination of eliminating unnecessary faceto-face encounters and increasing CVT visits also had the welcome benefit of reducing the clinic no-show rates. This further frees clinic appointments to accommodate additional patients and reduces the economic burden on the system ${ }^{24,25,29}$ and pa- tient wait times. As these are still newer processes, ${ }^{28}$ additional studies to specifically identify best practices to improve their use will be critical to improve efficiency in the system.

Implementing lean methods in a VA institution can be a complex undertaking, and for services new to the process, it is necessary to enlist guidance from experts in Lean Six Sigma. Our institution established a systems redesign service in the early part of the decade, and numerous departments have worked with it to effect change via lean methods in a cost-neutral manner. In the recent past, many authors have analyzed the factors that underlie the systematic inefficiencies that are inherent to the VA system. ${ }^{6-8}$ Some have even suggested wide-sweeping reforms that may involve a complete redesign of the system. ${ }^{8}$ The VA has long sought to improve its wait times, ${ }^{6,7,30,31}$ and this study demonstrated that a significant decrease in wait times is possible within the VA by analyzing systemic inefficiencies via lean methods, targeting wasteful processes for improvements, creating well-defined and realistic targets, and measuring outcomes of specific improvements. Not only is this approach far more costeffective than a complete system redesign but it also capitalizes on the inherent strengths of existing VA infrastructure.

This study has several limitations. First, the study only addressed a few factors that were monitored over 3 fiscal years. Additional years may need to be studied before long-term results can be validated. Furthermore, although there were no changes in the number of surgeons during the period of the study, 1 OR was shut down during 2014, resulting in the loss of block time. This confounded the results to some extent; however, operative volume remained higher than baseline from FY 2012. More importantly, access improvements were sustained for more than a year. Prospective and multi-VA center studies that study these and other end points will likely be needed to validate these results on a larger scale, but common patient electronic information systems and similar national database architecture make these easier than similar non-VA studies.

We learned several lessons that can be applied to any system that may seek to replicate these processes to eliminate waste and improve efficiency. Conducting a VSA is a lengthy, time-consuming process fraught with disagreement among 
participants. However, participants must be able to voice opinions on equal footing and without intimidation to ensure concerns and solutions can be vetted based on practicality. The work must be divided into small, feasible projects and triaged based on the effort involved (ie, JDIs, RPIWs, or projects) to achieve results quickly. The metrics to be evaluated should be easy to measure, and the desired outcomes should be realistic. Finally, participant buy-in is necessary for project success. Failures are to be expected as part of any reform, but studying the failures via Plan-Do-Study-Act methods can yield eventual and sustainable improvements.

\section{Conclusions}

Multidisciplinary collaboration using lean techniques can result in significant and sustained gains in multiple areas within the VA system. By reducing systemic inefficiencies, we achieved increased patient throughput, decreased wait lists, and improved patient access in a cost-neutral manner. This can translate into improved satisfaction for the patients as well as for physicians, residents, nurses, and nurse practitioners in the VA.

\section{ARTICLE INFORMATION}

Accepted for Publication: June 4, 2016.

Published Online: September 7, 2016.

doi:10.1001/jamasurg.2016.2808

Author Contributions: Drs Valsangkar and Eppstein had full access to all of the data in the study and take responsibility for the integrity of the data and the accuracy of the data analysis.

Concept and design: Eppstein.

Acquisition, analysis, or interpretation of data: All authors.

Drafting of the manuscript: Valsangkar, Eppstein,

Lawson.

Critical revision of the manuscript for important intellectual content: Eppstein, Taylor.

Statistical analysis: Valsangkar, Lawson.

Administrative, technical, or material support:

Taylor.

Study supervision: Eppstein.

Conflict of Interest Disclosures: None reported.

Funding/Support: This material is the result of work supported with resources and the use of facilities at the Richard L. Roudebush Veterans Affairs Medical Center.

Role of the Funder/Sponsor: The funder had no role in the design and conduct of the study; collection, management, analysis, and interpretation of the data; and preparation, review, or approval of the manuscript.

Disclaimer: The contents do not represent the views of the US Department of Veterans Affairs or the United States Government.

Previous Presentation: This work was presented at the 2016 Association of VA Surgeons; April 10, 2016; Virginia Beach, Virginia.

\section{REFERENCES}

1. National Center of Veterans Analysis and Statistics. Department of Veterans Affairs statistics at a glance. http://www.va.gov/vetdata/docs /Quickfacts/Homepage_slideshow_06_04_16.pdf. Accessed August 6, 2016.

2. US Department of Veterans Affairs. Selected Veterans Health Administration characteristics: FY2002 to FY2014. https://catalog.data.gov /dataset/selected-veterans-health-administration -characteristics-fy2002-to-fy2014. Accessed August 7, 2016.

3. Shulkin DJ. Beyond the VA crisis: becoming a high-performance network. N Engl J Med. 2016;374 (11):1003-1005.

4. Brewster AL, Cherlin EJ, Ndumele CD, et al. What works in readmissions reduction: how hospitals improve performance. Med Care. 2016;54 (6):600-607.

5. Nabors R. Issues impacting access to timely care at VA medical facilities. https://www.whitehouse .gov/sites/default/files/docs/va_review.pdf. Accessed April 17, 2016.

6. Kizer KW, Jha AK. Restoring trust in VA health care. N Engl J Med. 2014;371(4):295-297.

7. Giroir BP, Wilensky GR. Reforming the Veterans Health Administration: beyond palliation of symptoms. N Engl J Med. 2015;373(18):1693-1695.

8. Weeks WB, Auerbach D. A VA exit strategy. N Engl J Med. 2014;371(9):789-791.

9. House Committee On Veterans Affairs. The Veterans Access, Choice and Accountability Act of 2014 highlights. https://veterans. house.gov/the -veterans-access-choice-and-accountability-act-of -2014-highlights. Accessed April 17, 2016.

10. US Department of Veterans Affairs. Fact sheet: Veterans Access, Choice and Accountability Act of 2014 ("Choice Act"). http://www.va.gov/opa /choiceact/documents/choice-act-summary.pdf. Accessed April 17, 2016.

11. Veterans Access, Choice, and Accountability Act of 2014, 38 USC §113-146 (2014).

12. Commission on Care. Interim report of the Commission on Care. https://commissiononcare .sites.usa.gov/files/2015/12/Commission-on-Care Interim-Report_2015-11-23_FINAL.pdf. Accessed April 17, 2016.

13. Womack JP, Jones DT. Lean Thinking: Banish Waste and Create Wealth in Your Corporation, Revised and Updated. 1st ed. New York, NY: Simon \& Schuster; 2003.

14. Hung D, Gray C, Martinez M, Schmittdiel J, Harrison MI. Acceptance of lean redesigns in primary care: a contextual analysis [published online March 2, 2016]. Health Care Manage Rev. doi:10.1097/HMR.0000000000000106.

15. Agarwal S, Gallo JJ, Parashar A, et al. Impact of lean six sigma process improvement methodology on cardiac catheterization laboratory efficiency. Cardiovasc Revasc Med. 2016;17(2):95-101.

16. Moraros J, Lemstra M, Nwankwo C. Lean interventions in healthcare: do they actually work? a systematic literature review. Int J Qual Health Care. 2016;28(2):150-165.

17. Sugianto JZ, Stewart B, Ambruzs JM, et al. Applying the principles of lean production to gastrointestinal biopsy handling: from the factory floor to the anatomic pathology laboratory. Lab Med. 2015;46(3):259-264.
18. Duska LR, Mueller J, Lothamer H, Pelkofski EB, Novicoff WM. Lean methodology improves efficiency in outpatient academic gynecologic oncology clinics. Gynecol Oncol. 2015;138(3):707-711.

19. Ajami S, Ketabi S, Sadeghian A,

Saghaeinnejad-Isfahani S. Improving the medical records department processes by lean management. J Educ Health Promot. 2015;4:48

20. Mazzocato $P$, Savage $C$, Brommels $M$, Aronsson $\mathrm{H}$, Thor J. Lean thinking in healthcare: a realist review of the literature. Qual Saf Health Care. 2010; 19(5):376-382

21. Lean Enterprise Institute. What is lean? http://www .lean.org/WhatsLean/. Accessed April 17, 2016.

22. Fong T. An army of patients: the VA struggles with a growing population of veterans using its healthcare system as it works to boost quality and capacity. Mod Healthc. 2003;33(20):48-50, 62.

23. Brown SH, Lincoln MJ, Groen PJ, Kolodner RM. VistA: US Department of Veterans Affairs national-scale HIS. Int J Med Inform. 2003;69(2-3): 135-156.

24. Kheirkhah P, Feng Q, Travis LM, Tavakoli-Tabasi $\mathrm{S}$, Sharafkhaneh A. Prevalence, predictors and economic consequences of no-shows. BMC Health Serv Res. 2016;16:13.

25. Perron NJ, Dao MD, Kossovsky MP, et al. Reduction of missed appointments at an urban primary care clinic: a randomised controlled study. BMC Fam Pract. 2010;11:79.

26. VA Telehealth Service. Real-time clinic based video telehealth. http://www.telehealth.va.gov /real-time/. Accessed April 17, 2016.

27. US Veterans Health Administration. An audit of the home telehealth program. http://www.va.gov /oig/pubs/VAOIG-13-00716-101.pdf. Accessed April 16, 2016.

28. Kummerow Broman K, Vella MA, Tarpley JL, Dittus RS, Roumie CL. Identification of postoperative care amenable to telehealth. Surgery. 2016;160(2):264-271.

29. Junod Perron N, Dao MD, Righini NC, et al. Text-messaging versus telephone reminders to reduce missed appointments in an academic primary care clinic: a randomized controlled trial. BMC Health Serv Res. 2013;13:125.

30. Freedberg SJ Jr. A new formula for VA health care. Natl J (Wash). 1998;30(12):638-639.

31. Kizer KW, Dudley RA. Extreme makeover: transformation of the veterans health care system. Annu Rev Public Health. 2009;30:313-339. 